\title{
Energy-Efficient GPU L2 Cache Design Using Instruction-Level Data Locality Similarity
}

\author{
JINGWEIJIA TAN and KAIGE YAN, Jilin University \\ SHUAIWEN LEON SONG, Pacific Northwest National Laboratory \\ XIN FU, University of Houston
}

\begin{abstract}
This article presents a novel energy-efficient cache design for massively parallel, throughput-oriented architectures like GPUs. Unlike L1 data cache on modern GPUs, L2 cache shared by all of the streaming multiprocessors is not the primary performance bottleneck, but it does consume a large amount of chip energy. We observe that L2 cache is significantly underutilized by spending $95.6 \%$ of the time storing useless data. If such "dead time" on L2 is identified and reduced, L2's energy efficiency can be drastically improved. Fortunately, we discover that the SIMT programming model of GPUs provides a unique feature among threads: instruction-level data locality similarity, which can be used to accurately predict the data re-reference counts at L2 cache block level. We propose a simple design that leverages this Locality Similarity to build an energyefficient GPU L2 Cache, named LoSCache. Specifically, LoSCache uses the data locality information from a small group of cooperative thread arrays to dynamically predict the L2-level data re-reference counts of the remaining cooperative thread arrays. After that, specific L2 cache lines can be powered off if they are predicted to be "dead" after certain accesses. Experimental results on a wide range of applications demonstrate that our proposed design can significantly reduce the L2 cache energy by an average of $64 \%$ with only $0.5 \%$ performance loss. In addition, LoSCache is cost effective, independent of the scheduling policies, and compatible with the state-of-the-art L1 cache designs for additional energy savings.
\end{abstract}

CCS Concepts: • Computing methodologies $\rightarrow$ Graphics processors; • Computer systems organization $\rightarrow$ Single instruction, multiple data; $\bullet$ Hardware $\rightarrow$ Power estimation and optimization;

Additional Key Words and Phrases: GPU, cache, energy-efficiency, locality similarity

A preliminary version of this work appeared in Proceedings of the 2019 Design, Automation, and Test in Europe Conference and Exhibition (DATE'19) [20].

The work was supported in part by National Natural Science Foundation of China (NSFC) under grants 61802143 and 61902142; the Jilin Scientific and Technological Development Program under grants 20190701016GH, 20190201189JC, and 20200403130SF; and the Research Project by the Education Department of Jilin Province under grant JJKH20190159KJ. This research was also partially supported by U.S. National Science Foundation grants CCF-1619243, CCF-1537085(CAREER), and CCF-1900904, and the DOE CENATE project.

Authors' addresses: J. Tan (corresponding author), College of Computer Science and Technology, Jilin University, 2699 Qianjin Street, Changchun, Jilin, 130012, China; email: jtan@jlu.edu.cn; K. Yan, College of Communication Engineering, Jilin University, 5372 Nanhu Street, Changchun, Jilin, 130012, China; email: yankaige@jlu.edu.cn; S. L. Song, High Performance Computing Group, Pacific Northwest National Laboratory, Richland, WA; email: shuaiwen.song@pnnl.gov; X. Fu, Electrical and Computer Engineering Department, University of Houston, Houston, TX; email: xinfu8@central.uh.edu.

Permission to make digital or hard copies of all or part of this work for personal or classroom use is granted without fee provided that copies are not made or distributed for profit or commercial advantage and that copies bear this notice and the full citation on the first page. Copyrights for components of this work owned by others than the author(s) must be honored. Abstracting with credit is permitted. To copy otherwise, or republish, to post on servers or to redistribute to lists, requires prior specific permission and/or a fee. Request permissions from permissions@acm.org.

(C) 2020 Copyright held by the owner/author(s). Publication rights licensed to ACM.

1084-4309/2020/08-ART52 \$15.00

https://doi.org/10.1145/3408060 


\section{ACM Reference format:}

Jingweijia Tan, Kaige Yan, Shuaiwen Leon Song, and Xin Fu. 2020. Energy-Efficient GPU L2 Cache Design Using Instruction-Level Data Locality Similarity. ACM Trans. Des. Autom. Electron. Syst. 25, 6, Article 52 (August 2020), 18 pages.

https://doi.org/10.1145/3408060

\section{INTRODUCTION}

With the high computational throughput, modern GPUs are extensively applied for accelerating high performance computing (HPC) applications. On-chip caches are adopted to enhance data locality of GPU applications. However, recent studies [6, 13, 17, 23] have suggested that the cache hierarchy is not efficiently utilized in current GPUs. These works primarily focus on addressing the inefficiency of L1 cache due to its performance criticality. In contrast, with a much larger capacity and lower placement in the memory hierarchy, the GPU L2 cache is largely ignored in the previous works since it is not a primary performance bottleneck. However, as a large on-chip SRAM structure [12], inefficient utilization of L2 cache can significantly increase the overall GPU energy consumption.

To better understand L2 cache efficiency, we first analyze its utilization. Figure 1 depicts a request sequence for a cache line. In this example, the cache line starts to store useless data after the last request of a certain data. We refer to the time duration during which a cache line stores useless data as "dead time," labeled in Figure 1. This results in energy waste. For a wide range of GPU applications (Section 3), we observe that L2 cache lines spend an average of $95.6 \%$ of the execution time being dead. This indicates that the current GPU L2 cache is poorly utilized and consumes a significant portion of energy in storing data that will not be re-referenced again in the future.

To further identify the root cause of this phenomenon, we then characterize and analyze the data locality of GPU applications. We find that the majority of data sharing occurs at the intraCTA (i.e., cooperative thread arrays) level in the GPU. As the L2 cache is shared by different CTAs, the lacking of inter-CTA locality in GPU applications leaves the L2 cache ineffective for handling a high percentage of data accesses. By further analyzing the data re-reference counts for all L2 accesses, we observe that the majority of the L2 data has no or few future re-references. These findings suggest the inefficient utilization of L2 is caused by the data locality characteristics of the current GPU applications.

To reduce the unnecessary energy waste caused by L2 underutilization, we need to identify and significantly reduce these dead-time periods. In this article, we make a key observation: data locality in GPU applications has instruction-level similarity among threads due to the unique feature of the single instruction, multiple thread (SIMT) programming model. This finding can be used to accurately predict the data re-reference counts at L2 cache line level. With this information, L2 cache lines can be powered off during dead-time periods. Based on this idea, we propose to leverage the Locality Similarity to build an energy-efficient GPU L2 Cache (LoSCache) to reduce the unnecessary energy waste in L2 caused by its utilization inefficiency. Based on the locality similarity, it uses the information from a small group of CTAs to accurately predict the L2-level data re-reference counts of the remaining CTAs. Such locality prediction is conducted at data level instead of cache line level. After this, LoSCache powers off the cache lines that are predicted to be dead after certain accesses to conserve energy. Experimental results demonstrate that LoSCache can reduce the L2 cache energy by an average of $64 \%$ with only $0.5 \%$ performance degradation due to its high prediction accuracy. Our sensitivity studies also show that the effectiveness of LoSCache is independent of $\mathrm{L} 1$ cache designs and warp scheduling policies. To summarize, this work makes the following contributions: 


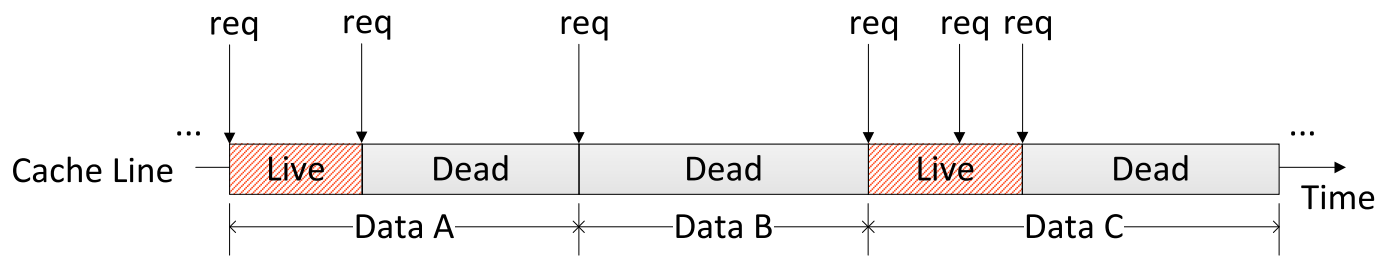

Fig. 1. Illustration for GPU L2 dead time. Dead time represents the time duration that stores "useless" data.

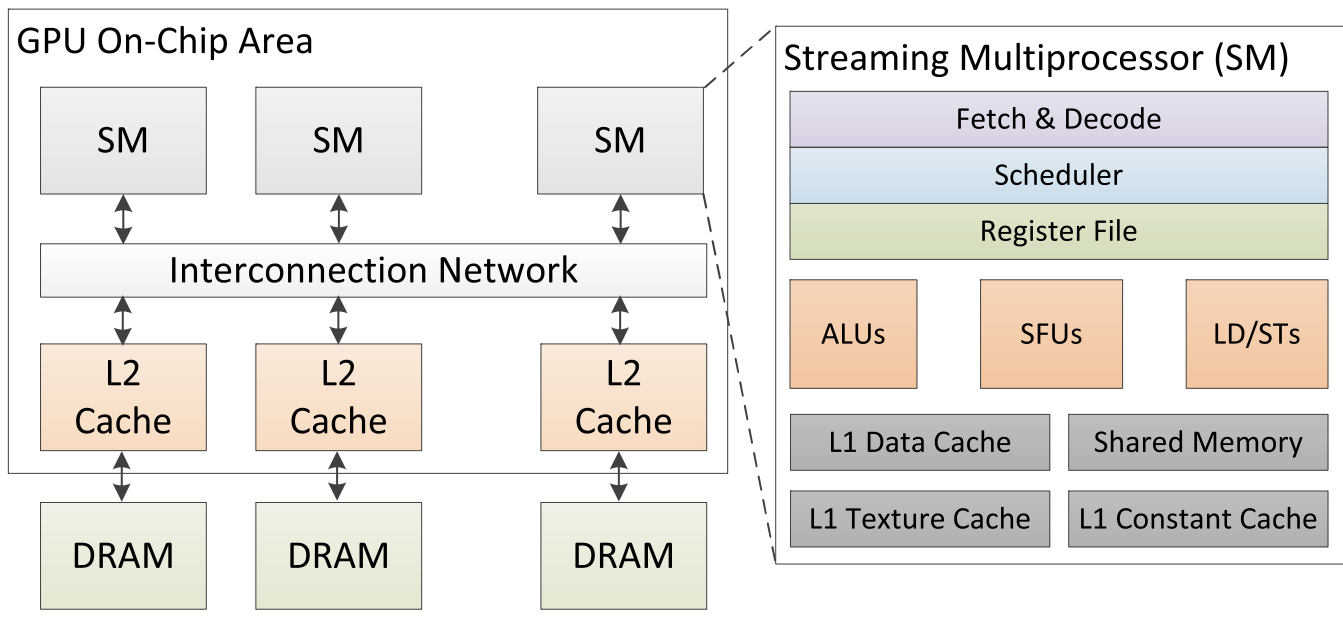

Fig. 2. Baseline GPU architecture.

- We find that GPU L2 cache lines store useless data for the majority of their lifetime due to the lack of inter-CTA locality in GPU applications. To the best of our knowledge, this is the first work to characterize and analyze data locality in L2 cache and demonstrate its inefficient utilization.

- We make a key observation that data locality of GPU applications has the instruction-level similarity among different threads due to the unique feature of the SIMT programming model. By leveraging this locality similarity, we can significantly reduce the energy waste at L2.

- We propose a design that is simple, cost effective, and light on resources. It is also compatible with different L1 cache designs for achieving further energy savings.

The rest of the article is organized as follows. We introduce the background of GPU architecture in Section 2. Section 3 details our observations for the instruction-level data locality similarity in L2 cache. We present our LoSCache design in Section 4. Section 5 introduces the experimental methodology, and Section 6 presents the experimental results. We discuss the related work in Section 7, and Section 8 concludes this work.

\section{BACKGROUND: GPU ARCHITECTURE}

Figure 2 shows the architecture of a modern GPU ${ }^{1}$. The GPU on-chip area is composed of a number of in-order streaming multiprocessors (SMs), multiple distributed L2 cache banks in different

\footnotetext{
${ }^{1}$ In this work, we focus on the NVIDIA CUDA [15] terminology to describe the GPU architecture and the programming model. Note that our work is independent of the terminology itself.
} 
memory partitions $[6,17,18]$, and an interconnection network (ICNT) that handles the communication among SMs and memory partitions. The right part of the figure shows a zoom-in view of an SM. It is composed of a scalar front-end that fetches and decodes the same instruction of a group of threads, and an SIMD back-end that executes the threads with different operands. The SIMD backend includes ALUs (which perform fundamental computation operations), SFUs (which perform fast approximate transcendental operations), and LD/ST units (which process memory accesses).

The GPU uses an SIMT programming model, which means threads within the same program kernel execute the same instruction with different thread operands. A kernel is composed of a grid of threads; a grid is divided into a set CTAs, or thread blocks. Each CTA is composed of hundreds of threads. Threads are distributed to SMs at the granularity of CTAs, and threads within a single CTA communicate via the shared memory and synchronize at a barrier if desired. The number of CTAs that execute concurrently in the same SM depends on their per-CTA resource requirements. Usually, not all CTAs from a program kernel can be allocated to the SMs in a single round. Thus, the remaining CTAs wait until their previous CTAs in the SMs finish and release their resources. In the SM pipeline, a number of threads from the same CTA are grouped together, called a warp. Threads within the same warp execute in SIMD mode, and the warp scheduler selects the ready warps for execution.

The caches in GPU are organized hierarchically, composed of SM-private L1 caches and a shared L2 cache. The L1 caches are only accessed by threads from the same SM, whereas the L2 cache is accessed by all threads of the kernel. The $\mathrm{L} 1$ data cache is typically write-evict and write no-allocate $[6,18]$, whereas the L2 cache is write-back with write-allocate $[6,18]$. To simplify the design, GPU caches are non-inclusive non-exclusive without hardware coherence $[6,18]$.

\section{MOTIVATION AND FINDINGS}

In this section, we first illustrate the utilization inefficiency of GPU L2 cache through profiling its cache line dead time for a wide range of applications. To further investigate what causes such long dead time for the L2 cache lines, we then characterize and analyze the intra-CTA and inter-CTA data locality. After that, we look into the distribution of the data re-reference and identify the root cause for GPU L2 inefficiency: only a small percentage of data has inter-CTA locality with a few re-references. Finally, we show our discovery for instruction-level data locality similarity on the GPU, which can be used to predict the data re-reference counts in L2 and eventually help address the energy waste issue by disabling the cache lines with long dead time.

\subsection{L2 Utilization Inefficiency}

Recall the illustration of cache line dead time in Figure 1; we want to investigate how much time GPU L2 cache lines spend on storing useless data. Figure 3 illustrates the dead-time percentage of L2 cache lines across different applications. ${ }^{2}$ The percentage value is calculated as Total_Dead_Time/(Execution_Time $\times$ Num_Cache_Lines). The figure clearly shows that on average, GPU L2 cache lines spend $95.6 \%$ of the time on storing data that will not be referenced again, indicating the inefficient utilization of GPU L2. Among all benchmarks, only MM has relatively lower (i.e., $<60 \%$ ) cache line dead time. This is because it well exploits the L2 capacity during execution. Note that several benchmarks (e.g., LIB, MM, NN) have larger footprints than the baseline L2 capacity, whereas others have very low L2 capacity utilization. We do not artificially change the L2 configuration nor tune the benchmarks to increase the L2 utilization to understand the actual characteristics of GPU L2 cache when executing different applications. To find out the root cause of this utilization inefficiency, we study the patterns of GPU data locality next.

\footnotetext{
${ }^{2}$ The detailed experimental methodology on the baseline architecture and benchmarks is introduced in Section 5.
} 


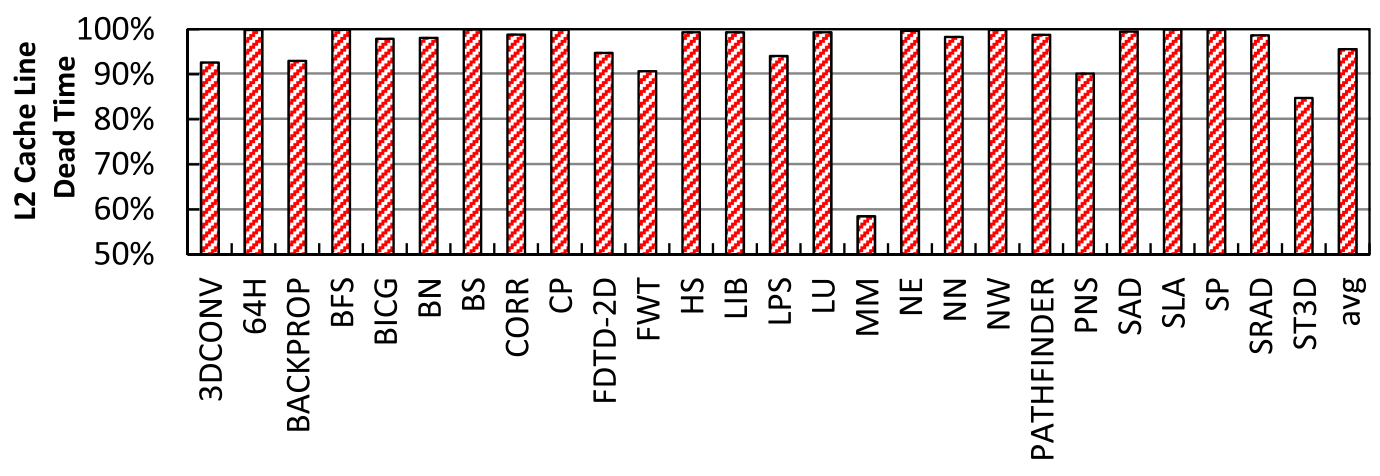

Fig. 3. L2 cache line dead time on the baseline architecture.

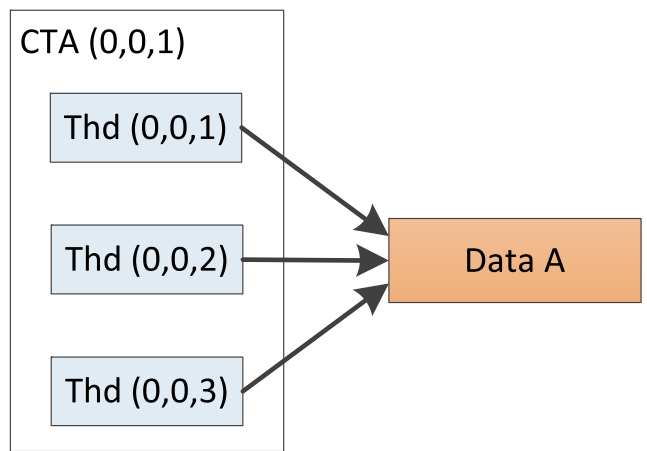

(a) Intra-CTA locality

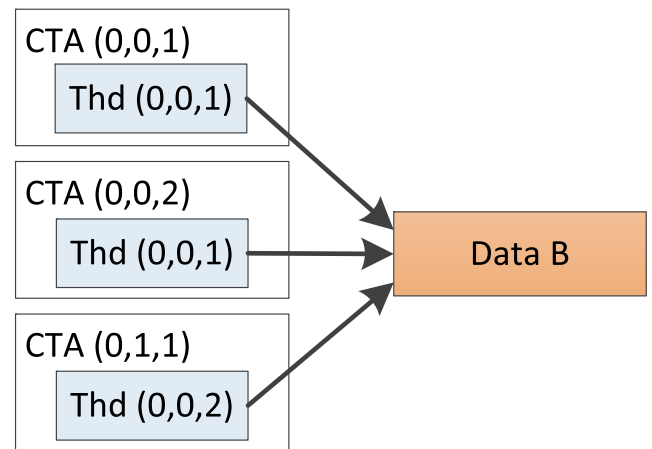

(b) Inter-CTA locality

Fig. 4. Examples of intra-CTA (a) and inter-CTA (b) data locality.

\subsection{Intra-CTA and Inter-CTA Locality}

As illustrated in Figure 2, L1 cache (private to each SM) and L2 cache (shared by all SMs) have different data preferences. In other words, data should be placed to its appropriate cache hierarchy to achieve the maximum performance benefit. Because the thread allocation, communication, and synchronization all occur at CTA level, we classify the locality of memory data into intra-CTA and inter-CTA locality. Figure 4 shows an example for these two locality cases. Intra-CTA locality (Figure 4(a)) describes the data that is only accessed by threads within the same CTA, which consists of both intra-warp locality (i.e., the data is only accessed by threads from the same warp) and inter-warp intra-CTA locality (i.e., the data is accessed by threads from different warps within the same CTA). Inter-CTA locality (Figure 4(b)) describes the scenario where data is accessed by threads from multiple CTAs. As threads within the same CTA execute on the same SM, ideally the per-SM L1 data cache is suitable to store the data with intra-CTA locality; these data should bypass the L2 cache to increase the cache capacity for data with inter-CTA locality.

To study the characteristics of GPU data locality, we first partition the GPU memory space into the same-sized chunks, each of which has the size of a cache line (e.g., 128B). We then check if each data chunk is accessed by memory requests from a single CTA or multiple CTAs in each benchmark. The data chunks that are only accessed by threads from a single CTA are considered as having intra-CTA locality, whereas the ones that are accessed by threads from multiple CTAs 


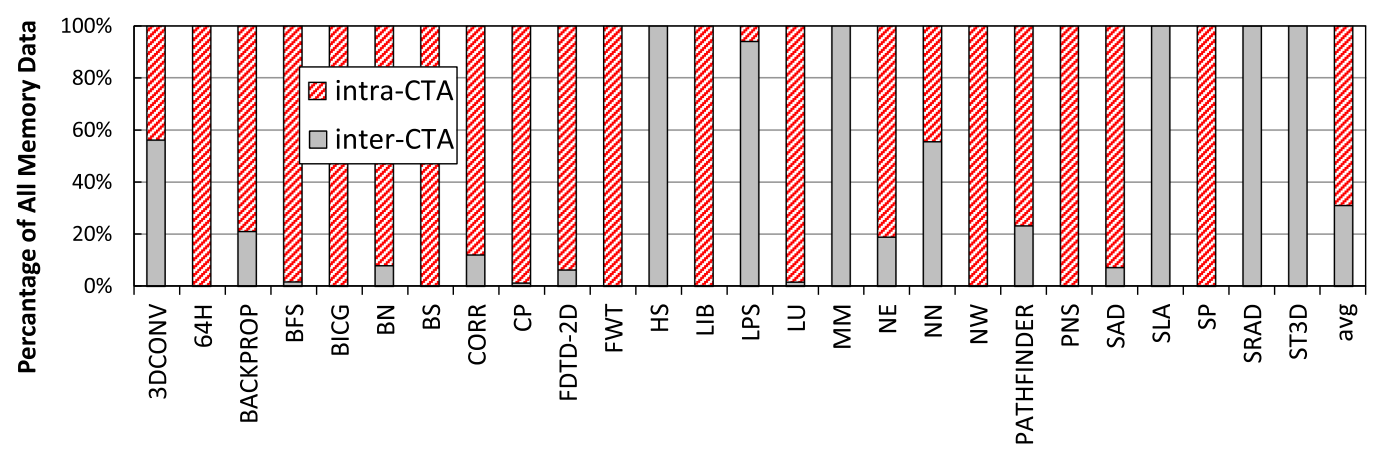

Fig. 5. Percentage of memory data (128B) with intra-CTA locality and inter-CTA, respectively.

are considered as having inter-CTA locality. Note that inter-CTA locality has two subcategories: inter-CTA locality on the same SM and across SMs. Figure 5 shows the percentage of data chunks with intra-CTA and inter-CTA locality for each application. As shown in this figure, the majority of memory data chucks have intra-CTA locality, accounting for $69 \%$ on average across all applications. In contrast, on average, only $31 \%$ memory data chunks have inter-CTA locality, indicating that some GPU applications tend not to share data among CTAs, which is consistent with the GPU programming model. Figure 5 also shows that 18 out of 26 benchmarks are dominated by intraCTA locality, except for 3DCONV, HS, LPS, MM, NN, SLA, SRAD, and ST3D, in which the majority of data exhibits inter-CTA locality. Among them, only 3DCONV has less than $1 \%$ of the data that has interCTA locality on the same SM due to the CTA scheduling. The rest of them do not contain data with inter-CTA locality on the same SM. Therefore, in this work, we only consider data having inter-CTA locality if it is accessed by CTAs across different SMs.

\subsection{Root Cause of L2 Utilization Inefficiency}

To explore the root cause of L2 utilization inefficiency, we further analyze the data re-reference distribution in L2 cache. The counting for data re-reference is conducted at L2 cache line level instead of evaluating individual memory access. We calculate the cache block address by masking the cache block offset of each memory access address. More specifically, we treat all accesses to the same L2 cache line address as accessing the same data, no matter if the actual data sizes of the requests are different or if the data has been evicted before being re-referenced. We then calculate the re-reference counts for the L2 data accesses in all benchmarks, and the results are shown in Figure 6 . Note that the analysis is based on the ideal L1 cache with unlimited capacity to analyze the real data result caused by inter-CTA locality. In this way, the reuse of intra-CTA locality in L2 is eliminated by avoiding the data thrashing in L1.

Figure 6 shows that most data accessed in L2 cache has low re-reference count due to the lack of inter-CTA locality. On average, $38 \%$ of the data will not be re-referenced again by the future L2 cache accesses, indicating that these data either have intra-CTA locality that has been captured by the L1 or no locality due to streaming (e.g., 64H, BICG, BS, and SP). Additionally, $27 \%$ of the data on average is re-referenced only once. Only $34 \%$ of the data in L2 cache is re-referenced more than once, the majority of which has inter-CTA locality. The rest of the data has intra-CTA locality but is referenced in L2. This is because data is written once or multiple times before it is read by the same CTA (i.e., as the L1 data cache is write-no-allocate in the GPU, data write will not be cached in L1 until the same data is read in the future), or thrashing occurs at L1. These observations from Figure 6 reveal the root cause of the L2 utilization inefficiency: the majority of the stored data in L2 has no or only one future re-reference, and even the data with inter-CTA locality still has 


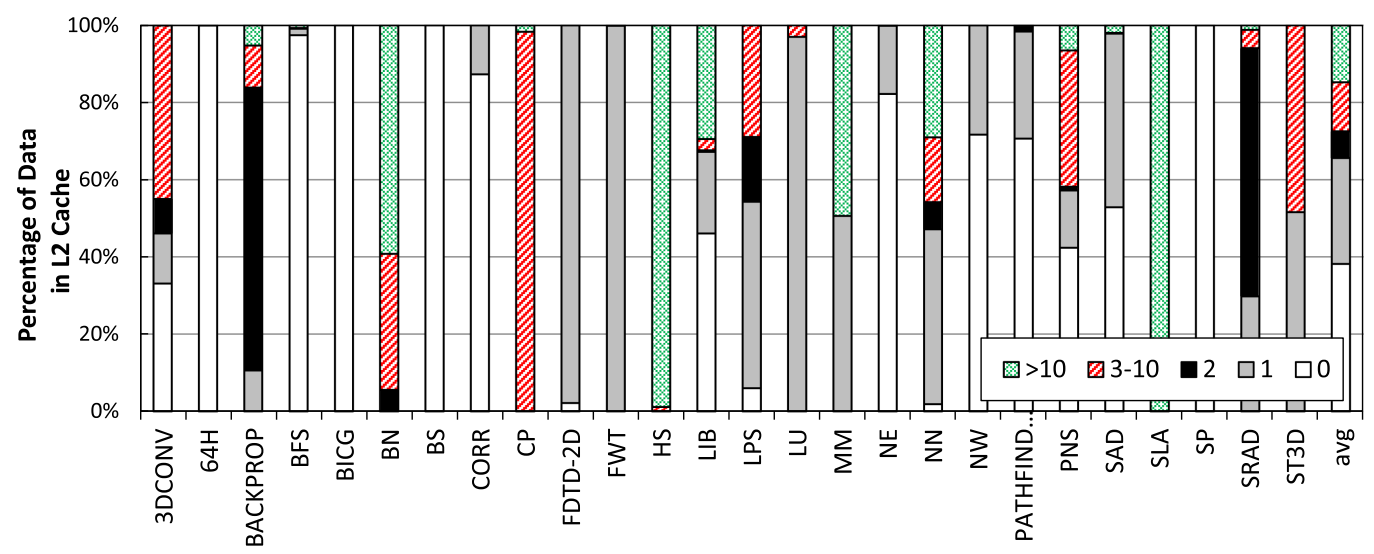

Fig. 6. Data re-reference counts in L2 cache under ideal L1 cache.

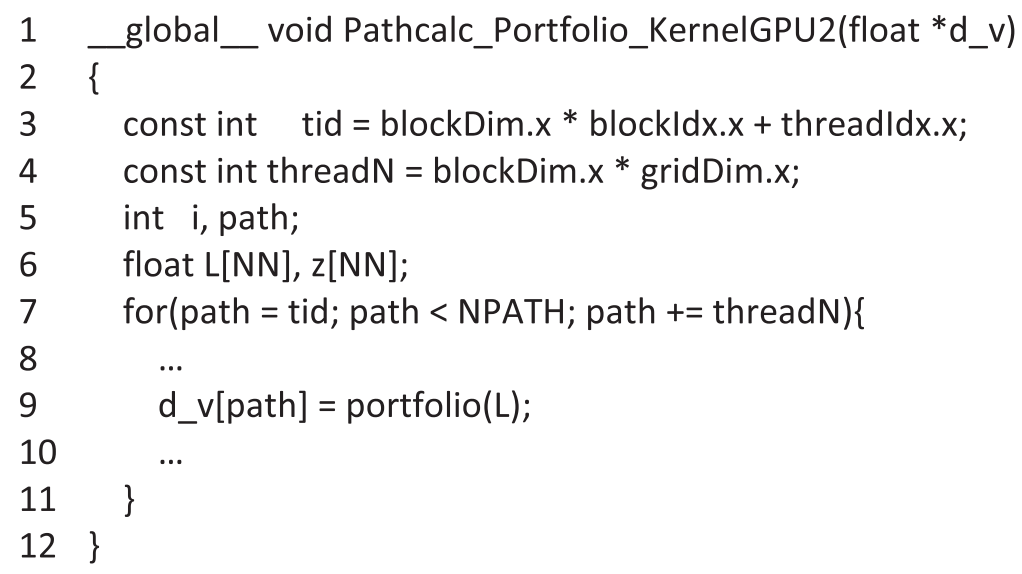

Fig. 7. Code example of LIB with intra-CTA locality.

relatively low future re-reference counts. This motivates us to design an energy-efficient L2 cache for the GPU: if the data re-reference counts can be predicted in advance, techniques such as power gating can be applied to shut off some cache lines during the time intervals in which they store useless data (or dead time shown in Figure 1).

\subsection{Instruction-Level Data Locality Similarity}

Since the GPU uses an SIMT programming model, all threads within the same kernel execute the same program code with different operands. Thus, when instructions with the same program counter (PC) are executed by different threads, they tend to exhibit similar behaviors. As a result, the data access requests generated by the same-PC instructions from different threads tend to have similar locality behavior as well. We explain the reason by analyzing the memory access instructions from two representative benchmarks: LIB and MM. By analyzing the data accesses, we observe that all accesses for LIB are intra-CTA locality, whereas more than $99 \%$ of the accesses for MM are inter-CTA locality. Figures 7 and 8 further illustrate two examples of data accesses from these two applications. For LIB shown in Figure 7, every thread loads data from the global memory to its own shared memory space (line 9) based on the CTA ID and thread ID (line 3). Since the 


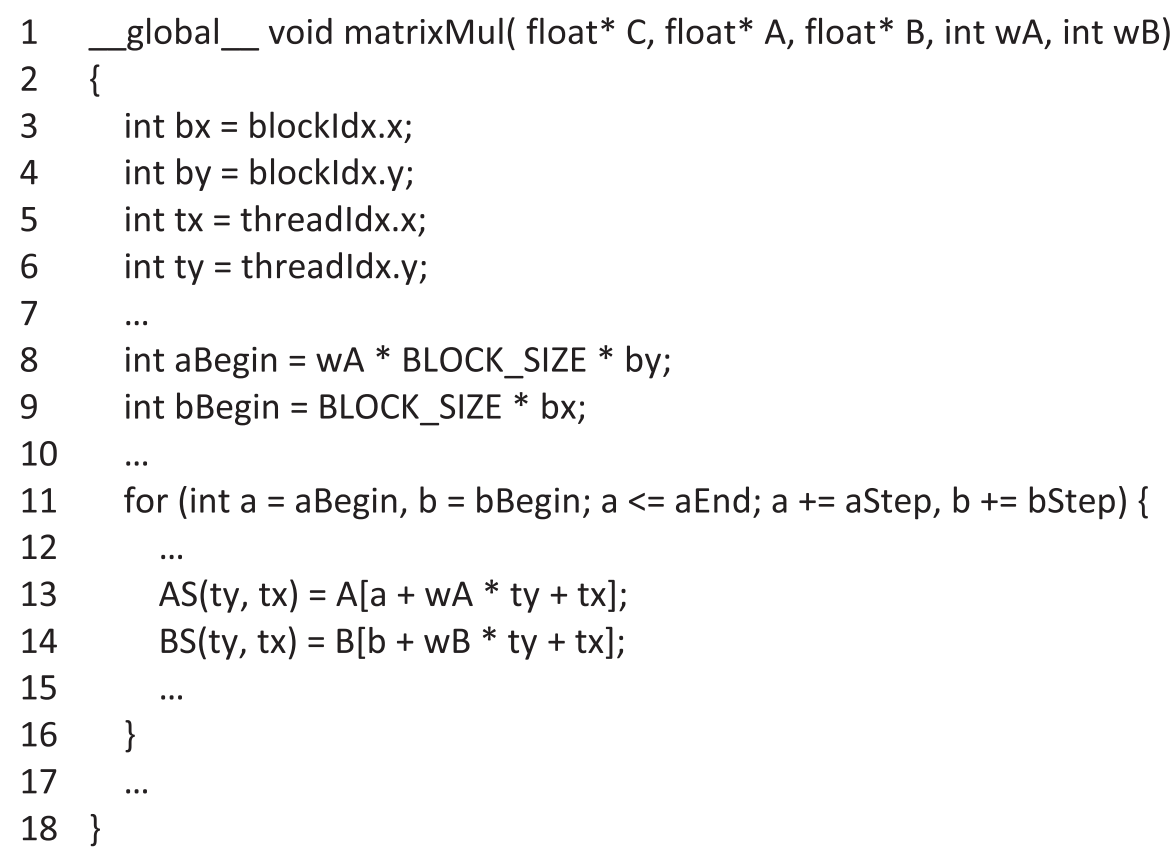

Fig. 8. Code example of MM with inter-CTA locality.

CTA ID and thread ID are both one dimensional in LIB, the data accesses have intra-CTA locality. Thus, even though different data is accessed by different threads when executing the same-PC instructions in this code, the data tends to show similar locality behavior.

Figure 8 shows the code example from MM. In this case, every thread loads the data from the global memory (i.e., A and B) to their own shared memory space (i.e., AS and BS) (lines 13 and 14) only based on the thread ID and one dimension of the block id (i.e., blockIdx.x or blockIdx.y) (lines 8 and 9). When executing the same instruction, threads from different CTAs access the same data from the global memory as long as their thread IDs and one dimension of their CTA ids are the same, resulting in inter-CTA locality. In addition, re-reference count in L2 for shared data is related to the number of CTAs that have the same dimension value. "Related" but not "equal" is because there are cases where CTAs with the same dimension value locate on the same SM, which does not belong to inter-CTA locality.

As shown in these examples, data locality in GPU applications has unique instruction-level similarity among different threads. We will leverage this opportunity to predict the data re-reference count next.

\section{DESIGN METHODOLOGY FOR LOSCACHE}

In this section, we propose a mechanism that leverages the instruction-level data locality similarity among SIMT threads to build an energy-efficient GPU L2 cache. First, we describe the prediction process for data re-reference counts. We then demonstrate how to leverage this prediction to design an energy-efficient GPU L2 cache, named LoSCache. Finally, we analyze the overhead of LoSCache. The goal of LoSCache is to gain the maximum energy savings from the dead time of L2 cache lines. 


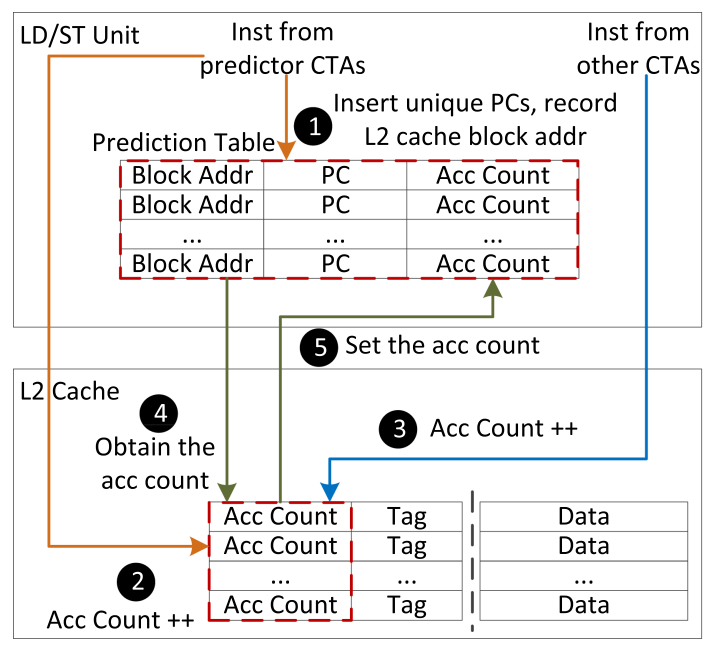

(a) Prediction Phase

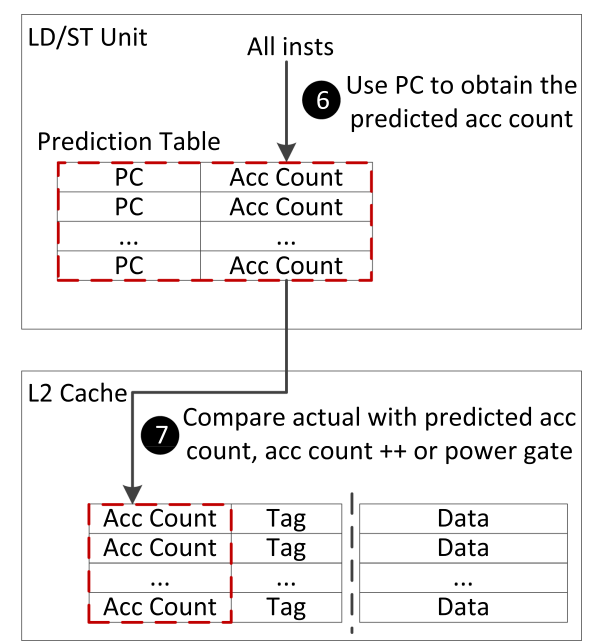

(b) Normal Execution Phase

Fig. 9. Architecture extensions and implementation of LoSCache. The red dashes (- - - ) locate the minor hardware extensions.

\subsection{LoSCache: Structure}

To implement LoSCache, we add minor hardware extensions to the LD/ST unit and L2 cache of the baseline GPU architecture, shown as the red-highlighted components in Figure 9. We discuss the structures of these two hardware extensions as follows.

Prediction table. Shown in Figure 9, a prediction table is placed in the LD/ST unit, which is aimed to predict the re-reference counts of data at L2 cache line level based on the instructionlevel data-locality similarity discussed previously. Some control logic are also added together with the prediction table. Each entry in the prediction table contains the locality prediction information about L2 cache accesses from instructions with an unique PC. Figure 9(a) shows the details of a prediction table entry, including the block address of the L2 cache line, the $P C$ of the instructions that access this L2 cache line, and the predicted access count to this cache line. The PC and the access count are used to indicate the predicted locality, whereas the block address is used to locate the corresponding L2 cache line at the end of the prediction phase (Section 4.2).

L2 cache extension. To assist the locality detection, we also extend the tag store of the L2 cache, shown in Figure 9. Each cache line is extended with an access count field to indicate the actual access count of the current data in this cache line. When the current data is evicted, the access count field is reset to 0 . This field is used to update the locality information of the prediction table at the end of the prediction phase and find the dead time of the corresponding cache line for energy savings during the normal execution. For power gating, a sleep transistor is added between Vdd and the circuit block of each cache line to control the power supply [3]. The sleep transistor is able to control the power supply for each individual cache line by turning on the cache line via ON mode while turning off a cache line via OFF mode.

\subsection{LoSCache: Operation}

The three major operation phases of LoSCache design are predictor selection, prediction phase, and normal excution, and are discussed next.

Predictor selection. During the program execution, different threads tend to execute in a similar pace on the GPU due to the SIMT model and hardware scheduling. In addition, the access interval 
to the same memory address is usually long because of the massive thread interleaving. Thus, it is difficult to predict the data locality of each memory request under the current scheduling policies (e.g., Greedy-Then-Oldest (GTO) [17]). Our basic idea to address this issue is to select some "predictors" and execute them faster to make runtime decisions for the others. A simple way to implement this on the GPU is to detect the locality information of all memory accesses in a single thread and use it to predict the locality for all other threads within the same kernel. However, an accurate prediction will not be reached until the predicting thread completes a large amount of the memory access instructions. In addition, the faster execution of this thread will be impeded by the CTA-level barriers. Additionally, the locality information of a single thread may be biased. In this work, we propose an efficient mechanism to cut down the drawbacks from single thread prediction. We randomly select one CTA from each SM as the predictor. This is because the characteristics of certain CTAs may be dramatically different from the others in the same application. For example, in one kernel of BFS, certain threads from a CTA are assigned with more workloads than the others, resulting in the imbalance of memory accesses among threads. Using the requests from one specific CTA to predict the other CTAs will be inaccurate. Our random predictor selection reduces the possibility of selecting monotonic CTAs as the predictors. Choosing multiple CTAs across SMs as predictors further reduces prediction bias as well.

Prediction phase. After the predictors are selected, the program goes through the prediction phase. During this phase, threads within the predictors have higher execution priority. Meanwhile, threads from the other CTAs execute in a lower priority; their ready threads will not execute until none of the threads in the predictors are ready (e.g., stalls due to data dependencies). In this way, the predictors can generate the locality information in advance for prediction. Figure 9(a) shows the prediction phase. When memory access instructions from the predictor CTAs are issued, their PCs and the L2 cache block addresses are inserted into the prediction table in parallel with the memory access request generation (1). The prediction table of each SM only stores entries with unique PCs. So before a PC is inserted, it will be first compared with the PCs of the existing entries in the same table. If there is a match, this PC will not be inserted. Meanwhile, the memory access requests are generated in $\mathrm{LD} / \mathrm{ST}$ units and sent to the $\mathrm{L} 2$ cache. The corresponding access count of the L2 cache line is increased by one from both the predictor CTAs (2) and the other normal CTAs (3). At the end of the prediction phase, the access count information of each entry in the prediction table is updated by using the block address of each entry to locate the corresponding L2 cache line (4), then updating the access count of the prediction table using the access count of the corresponding L2 cache line (5).

Normal execution. Once the prediction phase is completed, the information in the prediction table becomes available for future use. We refer to this phase as the normal execution phase, shown in Figure 9(b). During this phase, all memory request instructions check the prediction table using their instruction PCs to obtain the predicted access count of the corresponding L2 cache line (6). This is in parallel with the address calculation in the LD/ST unit. When accessing to the targeted data, the predicted access count is then compared with the current access count of the L2 cache line (7). If the current access count is smaller than the prediction, the current access count is increased by one. Otherwise, it means the access is the last one according to the prediction. The corresponding L2 cache line is then powered off after this access to save energy. If the predicted access count is one, the accessed data will not be stored in the L2 cache since by prediction it will not be referenced in L2. Note that all L2 cache lines are powered off at the beginning of the program and woken up when first accessed. This is because some applications are observed to only use a small percentage of L2 cache lines during the entire execution.

Figure 10 illustrates several prediction scenarios. For the baseline case (Figure 10(a)), six memory requests are sent to the same $\mathrm{L} 2$ cache line. The first four access data $\mathrm{A}$, and the last two access 


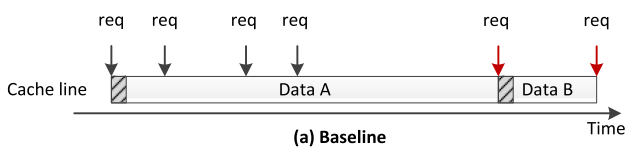

(a) Baseline

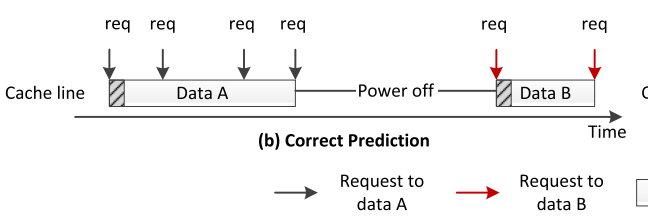

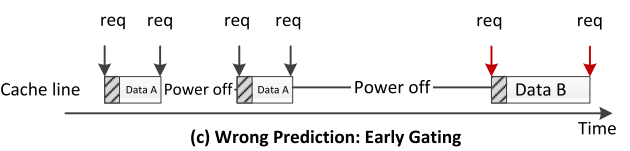

(c) Wrong Prediction: Early Gating

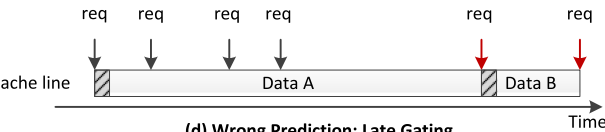

(d) Wrong Prediction: Late Gating

Reserved cache lin

Fig. 10. Prediction scenarios, including correct prediction, early gating, and late gating.

data $\mathrm{B}$. As this figure shows, the cache line contains useless data during the time between the last access to data A and the first access to data B. Under correct prediction (Figure 10(b)), the cache line is powered off during this time period to save energy without hurting the performance. If the predicted re-reference count is smaller than the actual re-reference count to the cache line for the entire program, the cache line is powered off earlier (i.e., early gating). As Figure 10(c) shows, it is powered off after the second request to data A. Thus, the third request becomes a miss, hurting the performance. Figure 10(d) shows the case of late gating, which means the predicted re-reference count is larger than the actual re-reference count of the data. In this case, the cache line will not be powered off during the time it contains useless data. As a consequence, there will be no energy savings (i.e., same as the baseline case).

\subsection{Optimizations}

Setting the prediction period. The prediction period is critical to the accuracy and effectiveness of the prediction. The most naive way is to terminate the prediction period when all predictor CTAs finish their execution. However, for applications that have a large amount of L2 accesses, this greatly reduces the effectiveness of the prediction since a decent prediction could very likely be available earlier than that time, limiting the energy-saving potential. Thus, we also consider L2 cache access intensity when determining the prediction period. Based on the experiments, we set $100 \mathrm{~L} 2$ cache accesses as the threshold. Therefore, the prediction period is finished after $100 \mathrm{~L} 2$ cache accesses or all predictor CTAs are completed, whichever comes first.

Increasing prediction accuracy. If the predicted re-reference count is smaller than the actual rereference count, the cache line is powered off earlier (i.e., early gating in Figure 10(c)). This case will increase the cache miss rate and hurt the performance. We apply an adaptive mechanism against this scenario. After a cache line is power gated, the tag still stores the information before the next data fills in. If the same data hits the tag of this cache line, it indicates that the actual re-reference count of the data is larger than the predicted. We then add a threshold for the future prediction of the accesses from this PC. In this case, the re-reference count for the future memory accesses is equal to the predicted value plus the threshold. Every inaccurate prediction will increase the threshold by one. Based on our experiments, a maximum threshold of three covers almost all applications we studied. Therefore, we set its upper bound to be three.

\subsection{Overhead Analysis}

Area overhead. The prediction table size is related to the number of unique PCs for the L 2 cache accesses during the prediction phase. Figure 11 shows the maximum number of unique PCs for each application. Based on the figure, NW has the largest number of unique PCs, which is 21. For each entry, we set 25 bits for the L2 block address, 32 bits for the PC field, and 6 bits for the 


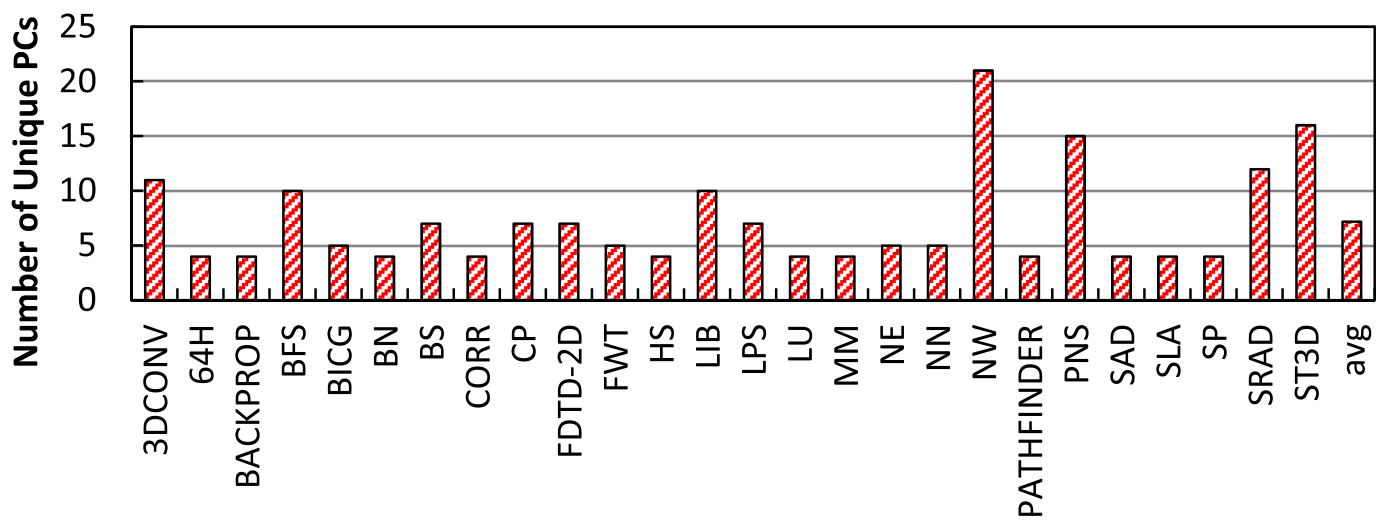

Fig. 11. Maximum number of unique PCs in the prediction table.

predicted access count (i.e., the highest L2 access count among all applications is 32). Therefore, we estimate the size of the prediction table as $21^{*}(25+32+6)=165$ Bytes per LD/ST unit. We set the access count in the L2 cache line to 6 bits, same as that in the prediction table. Thus, the storage overhead is 6 bits per L2 cache line. We use CACTI 6.5 [22] to calculate the area overhead of the added storage structures under 40-nm technology. For the control and gating logic, we apply a similar method used in the work of Rogers et al. [16] and Ran et al. [19] to aggressively estimate their area overhead as three times the added storage structures. We estimated that the total area overhead of the design is $0.449 \mathrm{~mm}^{2}$, only $0.084 \%$ of the entire GPU chip area.

Latency overhead. We assume that accessing the prediction table takes one additional cycle. However, this latency can be well hidden since it occurs in parallel with memory address generation in the LD/ST units. Additionally, there are also wake-up periods for the power-gated cache lines. Similarly, the wake-up process does not negatively affect the overall performance since it occurs in parallel with the data access to the lower level memory hierarchy. In other words, the cache line reservation periods require fetching of new data from the memory during cache misses anyway. Finally, at the end of the prediction phase, setting the access count in the prediction table may cause higher interconnect network traffic. We will analyze it in Section 6.2.

\section{EXPERIMENTAL METHODOLOGY}

Simulation environment. Our proposed LoSCache is evaluated using GPGPU-Sim V3.2.2 [4], a widely adopted cycle-accurate simulator for GPU architecture research. The configuration parameters of the baseline GPU architecture are shown in Table 1. We use a modified GPUWattch [12] to evaluate the dynamic and leakage energy consumption of LoSCache. We also include the energy consumption of accessing our hardware extensions in our evaluation (i.e., accessing the prediction table in the LD/ST unit and extended L2 tag).

Benchmarks. To faithfully evaluate our proposed technique, we collect a large set of 26 representative applications from the NVIDIA CUDA SDK [1], PolyBench [8], Rodinia benchmark suite [5], and Parboil benchmark suite [2], with default inputs. In our experiments, different kernels are executed sequentially (i.e., the following kernel will not be launched until the previous one finishes) and all workloads run to completion on the simulator.

\section{EXPERIMENTAL RESULTS AND ANALYSIS}

In this section, we first evaluate the prediction accuracy of LoSCache. We then analyze its impact on the ICNT traffic and the overall performance. After that, we explore the energy savings achieved 
Table 1. Baseline Architecture Configuration

\begin{tabular}{|l|l|}
\hline Parameters & \multicolumn{1}{|c|}{ Value } \\
\hline \hline SIMT Core (SM) & $\begin{array}{l}\text { 15 cores, SIMD width = 32, 1.4 GHz, } \\
\text { 5-stage pipeline }\end{array}$ \\
\hline Max/SM & $\begin{array}{l}\text { 1,536 threads, 32,768 registers, 48 warps, } \\
\text { 32 MSHRs with 256 entries }\end{array}$ \\
\hline L1 Cache/SM & $\begin{array}{l}16 \mathrm{~KB} / \text { core, 128B line, 4-way assoc, } \\
\text { 1-cycle hit latency }\end{array}$ \\
\hline Shared Mem/SM & $48 \mathrm{~KB}, 32$ banks, 3-cycle latency, \\
& 1 access per cycle \\
\hline Unified L2 Cache & $768 \mathrm{~KB}, 128 \mathrm{~KB} /$ bank, 6 banks, \\
& $128 \mathrm{~B}$ line, 16-way assoc \\
\hline DRAM & 6 memory channels, \\
& BW: 48 bytes/cycle, 1.4 GHz \\
\hline DRAM Schedule Queue & Size $=16$ and out of order (FR-RCFS) \\
\hline Warp Scheduling Policy & GTO \\
\hline
\end{tabular}

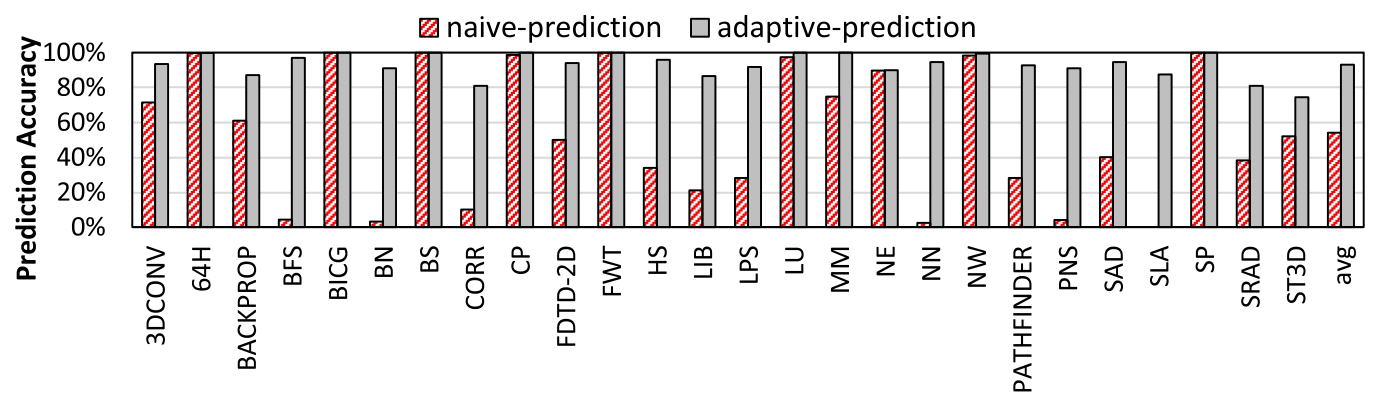

Fig. 12. Prediction accuracy comparison between naive- and adaptive-prediction.

by LoSCache. Finally, we conduct several sensitivity studies, including different L1 cache designs and warp scheduling policies, to evaluate the effectiveness of LoSCache under different design scenarios.

\subsection{Prediction Accuracy}

We first evaluate the prediction accuracy of LoSCache. Figure 12 shows the prediction accuracy for naive- and adaptive-prediction (introduced in Section 4.3 to increase prediction accuracy), respectively. The prediction accuracy indicates the percentage of correct predictions (i.e., predicted access count is the same as the actual access count) among all predictions. The figure shows that the prediction accuracy for naive-prediction is low, which is $54 \%$ on average. In contrast, adaptiveprediction greatly increases the prediction accuracy to $93 \%$ on average. Based on the accuracy evaluation standard, we check if the prediction is absolutely accurate for each data. For some cases, naive-prediction can only predict a similar locality but not the exact. However, by only adaptively increasing a small threshold, the prediction accuracy is significantly improved. For example, adding the threshold increases the prediction accuracy of BFS from $4 \%$ to $97 \%$. However, adaptive-prediction is unable to further increase the prediction accuracy for some benchmarks, including $64 \mathrm{H}, \mathrm{BICG}, \mathrm{BS}, \mathrm{CP}, \mathrm{FWT}, \mathrm{NE}$, and SP, since their prediction accuracy is already very high with naive-prediction. From this point forward, we use adaptive-prediction in LoSCache evaluation. 


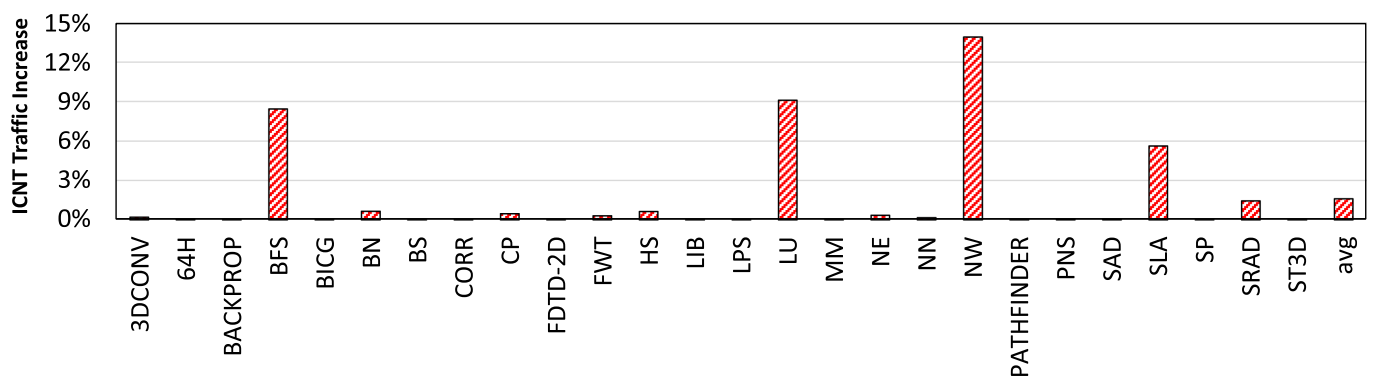

Fig. 13. Normalized ICNT traffic increase over the baseline architecture.

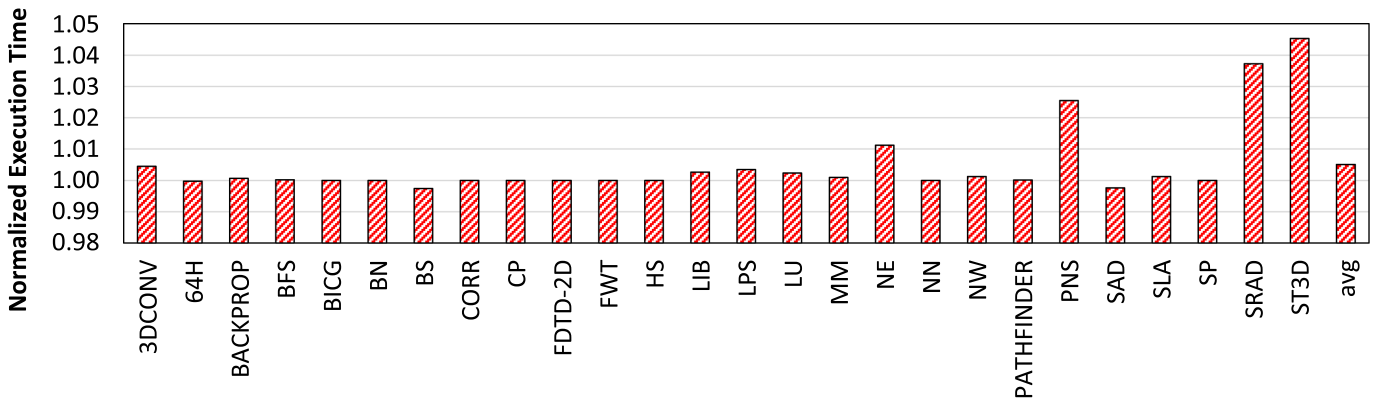

Fig. 14. Normalized execution time of LoSCache against the baseline architecture.

\subsection{ICNT Traffic Increase}

As discussed in Section 4.2, LoSCache uses the access counts of the L2 cache lines to set the access counts of the prediction table at the end of the prediction phase, which may result in an ICNT traffic increase. We further evaluate the ICNT traffic increase, which is shown in Figure 13. The average ICNT traffic increase is only $2 \%$ across all benchmarks. The reason for this negligible traffic increase is due to the small size of the prediction tables. The average number of valid entries (i.e., entries that contain active prediction information) in a prediction table is only 7 across all benchmarks (i.e., the average number of the unique PCs in a table is seven). In addition, LoSCache only updates the prediction tables at the end of the prediction phase. Some benchmarks, such as BFS, LU, NW, and SLA, encounter relatively higher ICNT traffic increase (i.e., $8 \%, 9 \%, 14 \%$, and $6 \%$, respectively) due to their relatively larger number of valid entries in the prediction tables (i.e., higher number of unique PCs) and small baseline ICNT traffic. For example, the number of valid entries in NW is 21, and it only generates several hundred interconnect network packages on the baseline architecture.

\subsection{Performance Overhead}

There are two potential reasons for LoSCache to incur performance overhead: (1) the slight ICNT traffic increase at the end of the prediction phase, which may degrade the performance, and (2) cache lines suffer from the early power gating due to inaccurate prediction, which increases the L2 miss rate. However, Figure 14 demonstrates that LoSCache design does not noticeably affect the overall performance. On average, the execution time only increases by $0.5 \%$. This result is consistent with the high prediction accuracy from our LoSCache design, shown in Figure 12. In addition, although the prediction accuracy for some benchmarks is lower, such as ST3D and SRAD, it only slightly impacts the performance. For example, the execution time of ST3D is only increased by $4 \%$ due to the small amount of early gating through incorrect prediction. Additionally, even 


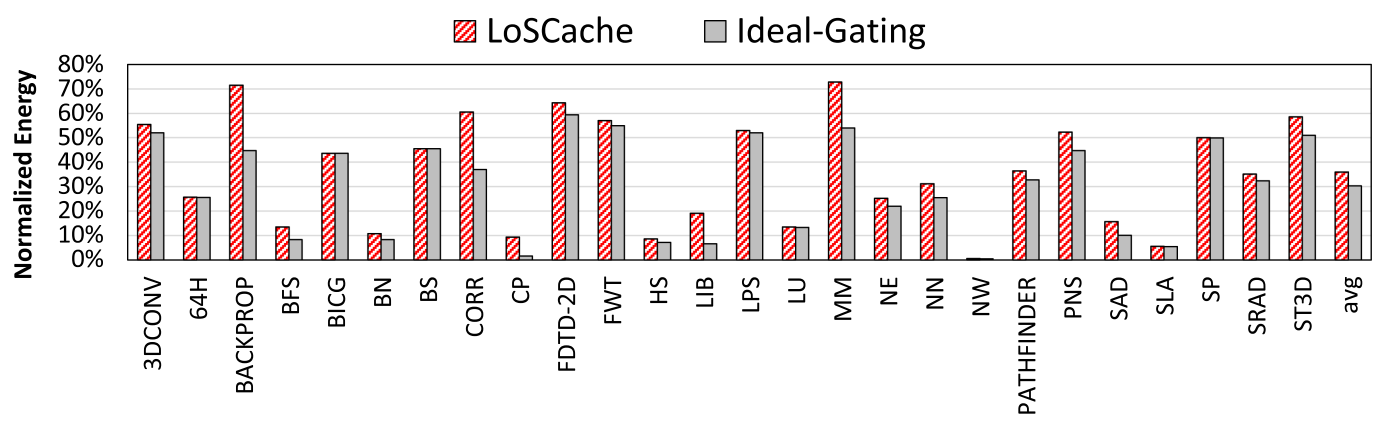

Fig. 15. Normalized energy consumption for LoSCache and Ideal-Gating against the baseline L2.

though NW has a $14 \%$ ICNT traffic increase, its performance degradation is less than $1 \%$. This is because its baseline ICNT traffic is so small that the traffic increase does not play a critical role in determining the overall performance.

\subsection{Energy Consumption}

We also evaluate the energy consumption under LoSCache. Figure 15 shows the normalized energy results against the baseline L2. As the figure shows, LoSCache greatly reduces the energy consumption from the baseline design. On average, the energy consumption is only $36 \%$ of the baseline scenario. LoSCache is effective for most benchmarks due to the low data re-reference counts in the L2 cache and its accurate prediction. For example, the energy consumption of NW is less than $1 \%$ of the baseline L2 cache due to its extremely low L2 cache utilization. However, LoSCache can only reduce the energy consumption of MM by $27 \%$ because of its high data re-reference counts. We also compare LoSCache with the Ideal-Gating technique, which is an oracle case that all L2 cache lines are powered off immediately once they expire. On average, Ideal-Gating further reduces L2 energy consumption by $6 \%$ compared to LoSCache. The reasons are twofold. Although the prediction accuracy of LoSCache is high, it is not $100 \%$. Thus, it wastes a small portion of energy on storing the expired cache lines due to the inaccurate prediction. The other reason is that LoSCache cannot power gate any expired cache line during the prediction phase. Thus, the additional energy savings achieved by Ideal-Gating is hard to achieve, and our LoSCache is already efficient enough. We further evaluate the energy savings of the entire GPU chip. Based on our evaluation, the energy consumption of GPU L2 cache is around 8\% of the total GPU chip energy. As a consequence, LoSCache reduces $6.2 \%$ of the total GPU chip energy on average.

\subsection{Sensitivity Analysis}

The impact of different $\mathrm{L} 1$ cache designs. We analyze the impact of different L1 cache designs on LoSCache via evaluating two extreme L1 cache designs: no L1 cache and ideal L1 cache (i.e., very large to capture all intra-CTA locality). Figure 16 shows the normalized energy consumption of L2 cache under these two schemes. To make the comparison more clear, we also include the results of LoSCache under the baseline L1 cache in the figure. Note that results in this figure are normalized to the baseline of each scheme (e.g., the result of LoSCache under no L1 case is normalized to the baseline L2 cache under no L1 cache). As it shows, LoSCache can greatly reduce the energy consumption under all three L1 cache designs. On average across all benchmarks, the L2 cache energy under LoSCache is reduced to $36 \%, 35 \%$, and $30 \%$ for baseline L1, no L1, and ideal L1, respectively. For many applications, including BACKPROP, BFS, MM, PATHF INDER, and PNS, LoSCache saves more energy under the ideal L1 cache design. This is because the ideal L1 cache design 


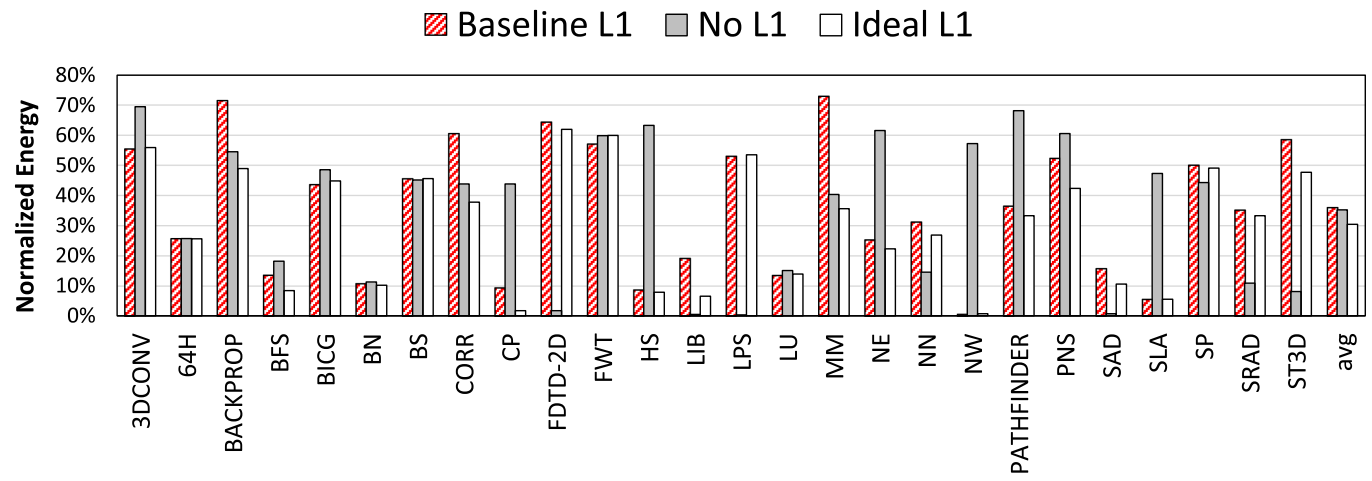

Fig. 16. Normalized energy of LoSCache under no L1 data cache and ideal L1 data cache, respectively.

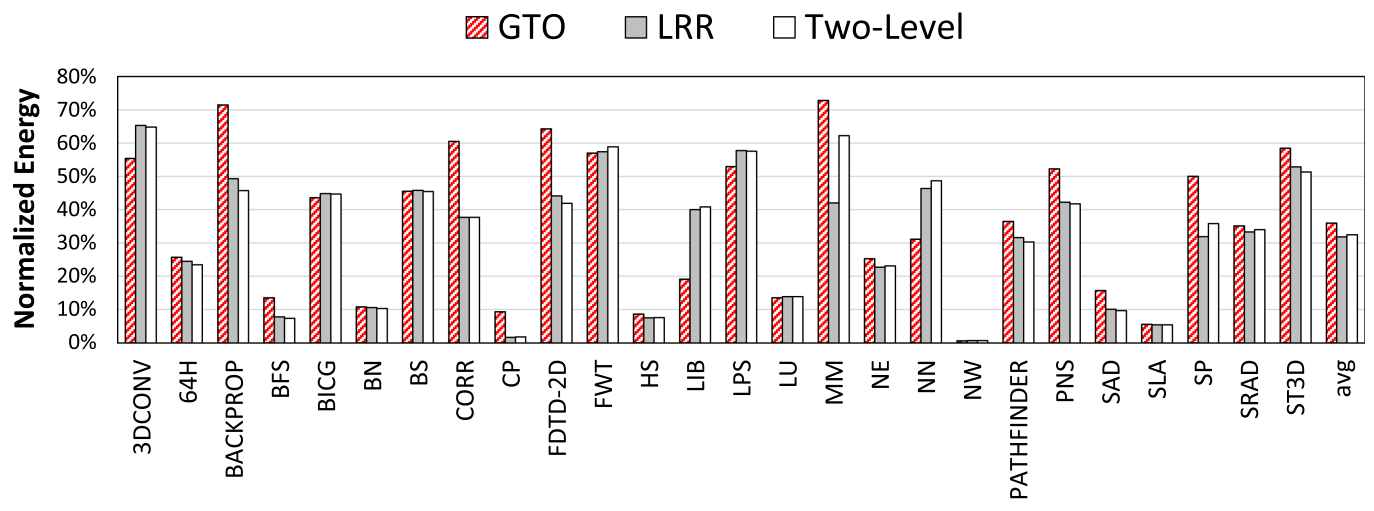

Fig. 17. Normalized energy of LoSCache under different scheduling policies.

reduces the L2 cache utilization, increasing energy-saving potential of LoSCache. We also observe some unconventional results, where the energy savings are larger for some benchmarks under no L1 design, including FDTD-2D, LIB, LPS, NN, SAD, SRAD, and ST3D. This is because LoSCache mainly reduces the leakage energy of the L2 cache. Under no L1 design, the total program execution time increases for applications with good L1 cache locality, and thus the leakage portion of the L2 cache energy increases. As a consequence, LoSCache under no L1 design saves more energy for these applications. Finally, these results confirm that our design is complementary to the state-of-the-art L1 cache designs and is able to further reduce energy.

The impact of scheduling policies. We further analyze the impact of different warp scheduling policies on LoSCache. Figure 17 shows the results of GTO [17], Loose-Round-Robin (LRR), and Two-Level [7] scheduling. As the figure shows, on average across all benchmarks, the energy consumption of L2 is reduced to $36 \%, 32 \%$, and $32 \%$, respectively. It indicates that the energy-saving ability of LoSCache remains significant under different scheduling policies. We also observe that LoSCache achieves slightly lower energy savings under GTO scheduling. This is because the the execution progress of different warps has the largest variation under GTO, degrading the prediction accuracy of LoSCache.

\section{RELATED WORK}

Due to the massive thread-level parallelism and the limited capacity, the GPU caches are not efficiently used. Several recent studies have addressed this problem to mitigate the performance loss 
due to cache conflicts or resource contention [6,13, 17, 23]. For example, Rogers et al. [17] proposed a cache-conscious wavefront scheduling to maintain the intra-CTA cache locality in L1 cache. Xie et al. [23] proposed to improve the cache performance via a coordinated static code analysis and dynamic cache bypassing. These techniques all effectively improve the L1 cache performance by exploiting data locality and avoiding thrashing. However, the L2 cache utilization in these designs remains low. In contrast, our proposed LoSCache technique addresses the inefficiency of the L2 cache in GPU by leveraging the instruction-level data locality similarity to reduce unnecessary energy waste. LoSCache is compatible with all of these L1 cache management techniques and can achieve further energy savings.

Several previous works were also proposed to reduce the energy consumption of CPU caches $[9-11,14]$. For example, Kaxiras et al. [10] proposed to power off a cache line if it is not accessed after a long period of time. For this technique, the prediction for cache line dead time is coarse grained and only based on the cache line itself. In contrast, our technique leverages the unique locality similarity of GPU programs to accurately predict the dead time in advance and powers off a cache line immediately after the last request of a data access. Additionally, several other works have been proposed to reduce the energy consumption of GPU caches. For example, Wang et al. [21] proposed to put the L1 and L2 caches into sleep mode when they are not active to save energy. This work aims to reduce energy when the entire cache is not active. In contrast, our LoSCache is able to reduce the L2 energy when it is active by predicting the cache line dead time.

\section{CONCLUSION}

L2 cache is underutilized in modern GPUs. It stores useless data for the majority of time, and as a result, data in L2 has no or few future re-references. To address this inefficient utilization of L2 cache, we propose LoSCache, a simple and effective energy-efficient GPU L2 cache design. LoSCache leverages the GPU's unique instruction-level data locality similarity caused by the SIMT programming model to predict the data re-reference counts in L2. Based on this prediction mechanism, LoSCache powers off the L2 cache lines if they are predicted to be dead after certain accesses. Experimental results show that LoSCache dramatically reduces the energy consumption of the GPU L2 cache with negligible performance loss. Our sensitivity studies also demonstrate that LoSCache is compatible with different state-of-the-art L1 cache designs and scheduling policies, achieving further energy savings.

\section{REFERENCES}

[1] NVIDIA. n.d. CUDA SDK. Retrieved July 31, 2020 from https://developer.nvidia.com/cuda-downloads.

[2] GitHub. n.d. Parboil Benchmark Suite. Retrieved July 31, 2020 from https://github.com/abduld/Parboil.

[3] M. Abdel-Majeed, D. Wong, and M. Annavaram. 2013. Warped gates: Gating aware scheduling and power gating for GPGPUs. In Proceedings of the 2013 46th Annual IEEE/ACM International Symposium on Microarchitecture (MICRO'13). 111-122.

[4] A. Bakhoda, G. L. Yuan, W. W. L. Fung, H. Wong, and T. M. Aamodt. 2009. Analyzing CUDA workloads using a detailed GPU simulator. In Proceedings of the 2009 IEEE International Symposium on Performance Analysis of Systems and Software. 163-174. DOI : https://doi.org/10.1109/ISPASS.2009.4919648

[5] S. Che, M. Boyer, J. Meng, D. Tarjan, J. W. Sheaffer, S. Lee, and K. Skadron. 2009. Rodinia: A benchmark suite for heterogeneous computing. In Proceedings of the 2009 IEEE International Symposium on Workload Characterization (IISWC'09). 44-54. DOI : https://doi.org/10.1109/IISWC.2009.5306797

[6] X. Chen, L. Chang, C. I. Rodrigues, J. Lv, Z. Wang, and W. Hwu. 2014. Adaptive cache management for energyefficient GPU computing. In Proceedings of the International Symposium on Microarchitecture. 343-355. DOI: https://doi.org/10.1109/MICRO.2014.11

[7] M. Gebhart, D. R. Johnson, D. Tarjan, S. W. Keckler, W. J. Dally, E. Lindholm, and K. Skadron. 2011. Energy-efficient mechanisms for managing thread context in throughput processors. In Proceedings of the 2011 38th Annual International Symposium on Computer Architecture (ISCA'11). 235-246. DOI : https://doi.org/10.1145/2000064.2000093 
[8] S. Grauer-Gray, L. Xu, R. Searles, S. Ayalasomayajula, and J. Cavazos. 2012. Auto-tuning a high-level language targeted to GPU codes. In Proceedings of the 2012 Conference on Innovative Parallel Computing (InPar'12). 1-10. DOI: https: //doi.org/10.1109/InPar.2012.6339595

[9] Zhigang Hu, S. Kaxiras, and M. Martonosi. 2003. Timekeeping techniques for predicting and optimizing memory behavior. In Proceedings of the 2003 IEEE International Solid-State Circuits Conference (ISSCC'03). Digest of Technical Papers.166-485 vol.1. DOI : https://doi.org/10.1109/ISSCC.2003.1234251

[10] S. Kaxiras, Zhigang Hu, and M. Martonosi. 2001. Cache decay: Exploiting generational behavior to reduce cache leakage power. In Proceedings of the 28th Annual International Symposium on Computer Architecture. $240-251$. DOI : https://doi.org/10.1109/ISCA.2001.937453

[11] An-Chow Lai, C. Fide, and B. Falsafi. 2001. Dead-block prediction and dead-block correlating prefetchers. In Proceedings of the 28th Annual International Symposium on Computer Architecture. 144-154. DOI : https://doi.org/10.1109/ ISCA.2001.937443

[12] Jingwen Leng, Tayler Hetherington, Ahmed ElTantawy, Syed Gilani, Nam Sung Kim, Tor M. Aamodt, and Vijay Janapa Reddi. 2013. GPUWattch: Enabling energy optimizations in GPGPUs. In Proceedings of the 40th Annual International Symposium on Computer Architecture (ISCA'13). ACM, New York, NY, 487-498. DOI : https://doi.org/10.1145/ 2485922.2485964

[13] D. Li, M. Rhu, D. R. Johnson, M. O’Connor, M. Erez, D. Burger, D. S. Fussell, and S. W. Redder. 2015. Priority-based cache allocation in throughput processors. In Proceedings of the 2015 IEEE 21st International Symposium on High Performance Computer Architecture (HPCA'15). 89-100. DOI : https://doi.org/10.1109/HPCA.2015.7056024

[14] H. Liu, M. Ferdman, J. Huh, and D. Burger. 2008. Cache bursts: A new approach for eliminating dead blocks and increasing cache efficiency. In Proceedings of the 2008 41st IEEE/ACM International Symposium on Microarchitecture. 222-233. DOI : https://doi.org/10.1109/MICRO.2008.4771793

[15] NVIDIA. n.d. CUDA C Programming Guide. Retrieved July 31, 2020 from http://docs.nvidia.com/cuda/cuda-cprogramming-guidel

[16] T. G. Rogers, D. R. Johnson, M. O’Connor, and S. W. Keckler. 2015. A variable warp size architecture. In Proceedings of the 2015 ACM/IEEE 42nd Annual International Symposium on Computer Architecture (ISCA'15). 489-501. DOI : https: //doi.org/10.1145/2749469.2750410

[17] T. G. Rogers, M. O’Connor, and T. M. Aamodt. 2012. Cache-conscious wavefront scheduling. In Proceedings of the 2012 45th Annual IEEE/ACM International Symposium on Microarchitecture. 72-83. DOI : https://doi.org/10.1109/MICRO. 2012.16

[18] I. Singh, A. Shriraman, W. W. L. Fung, M. O'Connor, and T. M. Aamodt. 2013. Cache coherence for GPU architectures. In Proceedings of the 2013 IEEE 19th International Symposium on High Performance Computer Architecture (HPCA'13). 578-590. DOI : https://doi.org/10.1109/HPCA.2013.6522351

[19] J. Tan, S. L. Song, K. Yan, X. Fu, A. Marquez, and D. Kerbyson. 2016. Combating the reliability challenge of GPU register file at low supply voltage. In Proceedings of the 2016 International Conference on Parallel Architecture and Compilation Techniques (PACT'16). 3-15. DOI : https://doi.org/10.1145/2967938.2967951

[20] J. Tan, K. Yan, S. L. Song, and X. Fu. 2019. LoSCache: Leveraging locality similarity to build energy-efficient GPU L2 cache. In Proceedings of the 2019 Design, Automation, and Test in Europe Conference and Exhibition (DATE'19). 1190-1195. DOI : https://doi.org/10.23919/DATE.2019.8714911

[21] Y. Wang, S. Roy, and N. Ranganathan. 2012. Run-time power-gating in caches of GPUs for leakage energy savings. In Proceedings of the 2012 Design, Automation, and Test in Europe Conference and Exhibition (DATE'12). 300-303. DOI : https://doi.org/10.1109/DATE.2012.6176483

[22] S. J. E. Wilton and N. P. Jouppi. 1996. CACTI: An enhanced cache access and cycle time model. IEEE fournal of SolidState Circuits 31, 5 (May 1996), 677-688. DOI : https://doi.org/10.1109/4.509850

[23] X. Xie, Y. Liang, Y. Wang, G. Sun, and T. Wang. 2015. Coordinated static and dynamic cache bypassing for GPUs. In Proceedings of the 2015 IEEE 21st International Symposium on High Performance Computer Architecture (HPCA'15). 76-88. DOI : https://doi.org/10.1109/HPCA.2015.7056023

Received November 2019; revised May 2020; accepted June 2020 